\title{
On stress-forecasting strategy of earthquakes from stress buildup, stress shadow and stress transfer (SSS) based on numerical approach*
}

\author{
Chunan Tang ${ }^{1, \uparrow}$ Tianhui $\mathrm{Ma}^{1}$ and Xiaoli Ding ${ }^{2}$ \\ ${ }^{1}$ Center for Rock Instability and Seismicity Research, Northeastern University, Shenyang, China \\ ${ }^{2}$ Department of Land Surveying and Geo-Informatics, Hong Kong Polytechnic University, \\ Kowloon, Hong Kong, China
}

\begin{abstract}
Global Positioning System (GPS) and Interferometric Synthetic Aperture Radar (InSAR), used for monitoring crust deformation, are found to be very promising in earthquake prediction subject to stress-forecasting. However, it is recognized that unless we can give reasonable explanations of these curious precursory phenomena that continue to be serendipitously observed from time to time, such high technology of GPS or InSAR is difficult to be efficiently used. Therefore, a proper model revealing the relation between earthquake evolution and stress variation, such as the phenomena of stress buildup, stress shadow and stress transfer (SSS), is crucial to the GPS or InSAR based earthquake prediction. Here we address this question through a numerical approach of earthquake development using an intuitive physical model with a map-like configuration of discontinuous fault system. The simulation provides a physical basis for the principle of stress-forecasting of earthquakes based on SSS and for the application of GPS or InSAR in earthquake prediction. The observed SSS associated phenomena with images of stress distribution during the failure process can be continuously simulated. It is shown that the SSS are better indicators of earthquake precursors than that of seismic foreshocks, suggesting a predictability of earthquakes based on stress-forecasting strategy.
\end{abstract}

Key words: stress-forecasting; earthquake; stress buildup; stress shadow; stress transfer CLC number: P315.75 Document code: A

\section{Introduction}

An earthquake may be triggered when the stress buildup reaches the strength of the corresponding fault. During an earthquake, a stress shadow may be produced in the static stress field that may inhibit earthquakes and create a period of relative quiescence (Harris and Simpson, 1996) until the stresses have recovered due to the tectonic loading. In occasions, earthquakes often trigger other earthquakes or active seismic events on nearby faults (Scholz, 1999) due to the stress transfer from the stress released shadow region. In all the cases, the stress state and its variation, i.e., stress buildup, stress shadow and stress transfer (SSS), are the most fundamental physical parameters controlling the earthquake process

\footnotetext{
${ }^{*}$ Received 20 June 2008; accepted in revised form 10 September 2008; published 10 February 2009.

•Corresponding author. e-mail: tca@mail.neu.edu.cn
}

(Deng and Sykes, 1997). Nowadays, the SSS phenomena can be possibly monitored by the global positioning system (GPS) and interferometric synthetic aperture radar (InSAR) system (Peltzer et al, 1999, Relinger et al, 2000, Chen et al, 2000), which is believed to be very promising in its application in earthquake prediction based on the stress-forecasting principle, an apparently viable third strategy of earthquake prediction proposed by Crampin (1999) in a recent Nature debate (1999) and were addressed at a two-day workshop (Harris, 1998) on "stress triggers, stress shadows, and implications for seismic hazard". The significance of such a stress evolution modeling has also been demonstrated by Deng and Sykes (1997) in their case study on the stress changes in 200 years in the southern California since 1812. However, the theoretical studies of stress field evolution have been made difficult by lacking a suitable method to model the rock rupture process including the pre- and post-failure behavior. Laboratory rock friction experi- 
ments have elucidated the properties of smooth faults, but seem insufficient to explain the observed complexity of real fault dynamics (Heimpel, 1997). The past failures in earthquake predication also reflect that the widely accepted elastic rebound theory formulated by Brace and Byerlee (1966) is too simple: the natural faults are not as simple as in the Reid's model or the friction law model (Scholz, 1998). Complicated geometrical discontinuities in faults, such as branches, bends, and steps, are commonly observed at the Earth's surface by geological field investigators and they may control how an earthquake developed (Harris 1999). In many cases, the earthquakes may not be caused by the sudden slip of a fault as described by fiction law model, but by the sudden breakage of the fault bridge or step between two or more overlapped faults as in a en echelon fault system, such as the 1992 Landers earthquake (Harris and Day, 1999). Fault inflexion point is another important earthquake candidate site that cannot be modeled by the simple friction law. The past attempts to understand SSS are dominated by case studies. At an early stage these approaches can be valuable, but there are now literally thousands of published claims of case studies, and the value of further additions to this list is questionable. In addition, SSS have so far been treated separately and attempts trying to unify the requirements of SSS in a universal model are unfortunately rare. To accomplish such a task, a numerical code, RFPA ${ }^{2 D}$ (Rock Failure Process Analysis), has been developed by Dalian Mechsoft Co., Ltd (Mechanics Software). With the RFPA $^{2 \mathrm{D}}$ the stress distribution in failure process of crust model with a map-like configuration of discontinuous fault system (Harris 1999) can be continuously simulated. We are therefore able to study the pre- and post-failure behavior during a model earthquake. As a result, the phenomena of SSS, constituting the basis for the stress-forecasting strategy of earthquake prediction, can be numerically demonstrated in a spatial-temporal manner, while such phenomena would normally be observed only in a natural earthquake cycle that last for hundreds or thousands of years.

\section{Numerical procedure}

We use a map-view cross section with a number of faults to represent a model seismogenic area in the crust. The 2D model is composed of a fault zone system embedded in a heterogeneous elastic medium under tectonic loading. To simulate the heterogeneity of the matrix material we use an elastic modulus $E$, and yield strength, $\sigma_{c}$, that is randomly drawn from a Weibul's distribution (Tang, 1997) through Monte-Carlo method. A plane stress condition for the entire models is postulated. Inertia is neglected (quasi-static approximation).

As illustrated in Figure 1, the model consists of $250 \times 200$ square elements. Ten discontinuous faults marked with " $a$ "-" $j "$ are setup in the model along the diagonal from upper left corner to bottom right corner with an arbitrary shape, length and orientation, resembling the natural geographical locations of faults or sub-faults (Harris and Day, 1999). The mechanical property of the fault material is assumed as ten times weaker than that of the crust material. As boundary conditions, a constant displacement increment rate is imposed on the vertical model boundaries and a constant compression is applied normal to the side boundaries, to model the far field tectonic loading. A model shock occurs when the strength of an element reaches the yield strength, resulting in an elastic energy release at that element. Because breakage of the element changes the stress field around it, one such breakage may precipitate a large shock as a cascade of many individual broken elements. For every shock each numerical element that breaks at least once is given a residual strength. The new residual strength value is retained for subsequent breakage during its shock. This ad hoc rule (Heimpel, 1997) is meant to represent brittle failure of fault gouge and crust mediums, where the strength randomness represents the local heterogeneity associated with fault asperities and damaged crust mediums.

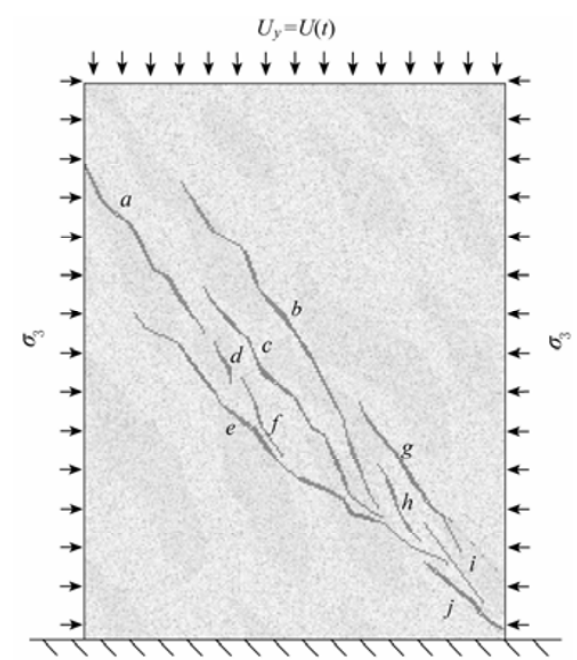

Figure 1 RFPA $^{2 D}$ model and its boundary conditions with ten faults distributed in a diagonal zone. The faults are marked with " $a$ " to " $j$ ". 


\section{Seismic cycles and energy release}

The numerically obtained relations between seismic cycles and energy release are illustrated in Figure 2. In Figure 2a, axial stress is plotted as a function of time step (proportional to axial displacement). The simulated time evolution curves of seismic events and associated energy release are shown in Figures $2 b$ and 2c. In contrast to most of the conceptual models of earthquakes, such as the lattice network models (Marder and Liu, 1993; Abraham et al, 1994), the granular models (Scott, 1996; Liu et al, 1994), the electrical resistor network model (Zapperi et al, 1997), the cellular automation model (Sammis and Smith, 1999b), or fractal model (Huang et al, 1998), our model reproduces several more realistic observed seismic patterns of earthquakes: large shocks are preceded by localized foreshocks, and followed by aftershock sequences, which constitutes a complete sequence of earthquake cycles. Here a seismic cycle consists of a period of accelerating seismic release, culminating in one or more large shocks, and a period of decelerating seismic release (Bufe and Varnes, 1993). As pointed out by Bufe and Varnes (1993) that the long-term seismic cycle for the largest earthquake in a region can be modeled as the sum of a series of subcycles. In our model, two of such subcycles are simulated (shown by the dashed lines in Figure 2c). We find that each seismic cycle consists of a complete sequence of a period of accelerating seismic release, culminating in one large shock, and a period of decelerating seismic release. It is important to note that, in the first cycle, the pattern of increasing seismicity was interrupted by a period of relative quiescence between the 106-115 loading steps, shown in Figure 2c, which ended in the 115 step with the occurrence of the mainshock of $M 1(0 \leq M \leq 1$ is the normalized magnitude), which is similar to the seismic pattern of the Coyote Lake earthquake in northern California in 1979 (Bufe and Varnes, 1993). It is also found that following the aftershock sequence of the first cycle, there is a relative long time quiescence of seismic activity until the next $M 0.73$ mainshock (step 166) occurred with much lower magnitude foreshocks. The seismic pattern that the second mainshock has a much lower magnitude foreshock pattern than that of the first one was found by Mogi (1969) in his studies of earthquakes in Japan, and has been proved by many case studies (Mogi, 1969; Jaumé and Sykes, 1999).
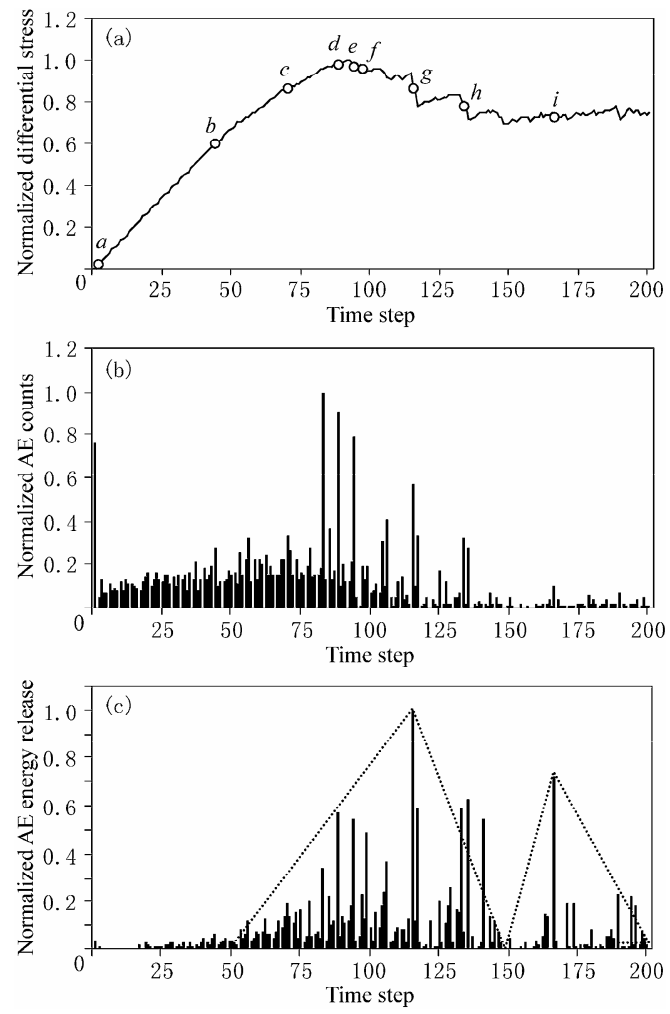

Figure 2 Simulated stress (a), AE counts (b) and AE released energy (c) plotted against time step (proportional to displacement). Letters " $a$ "-" $i$ " along stress curve correspond to plots shown in Figure 3. The two dashed triangles represent two earthquake cycles.

\section{Fault developments inferred from seismic locations}

Figure 3 shows the locations of seismic events in nine sequential time intervals that correspond to the stress intervals indicated in Figure 2a. Each circle represents one event or shock and the diameter of the circle represents the relative magnitude of the released energy. Figure 3a shows that the relatively diffuse seismic events occur during the beginning of loading. From Figure $3 \mathrm{~b}$ to Figure $3 \mathrm{i}$, it is clearly seen that the seismic events tend to be localized in a well-defined seismic activity belt. A comparison between the seismic event map (Figure 3) and the fault geometry in the original model (Figure 1) shows that most of the moderate to large seismic events occur in the region within the fault system.

Theoretically, recognizing foreshocks would allow us to predict mainshocks. However, in earthquake prediction, as many others found (Jaumé and Sykes, 1999), no one has been able to easily identify which shocks are 

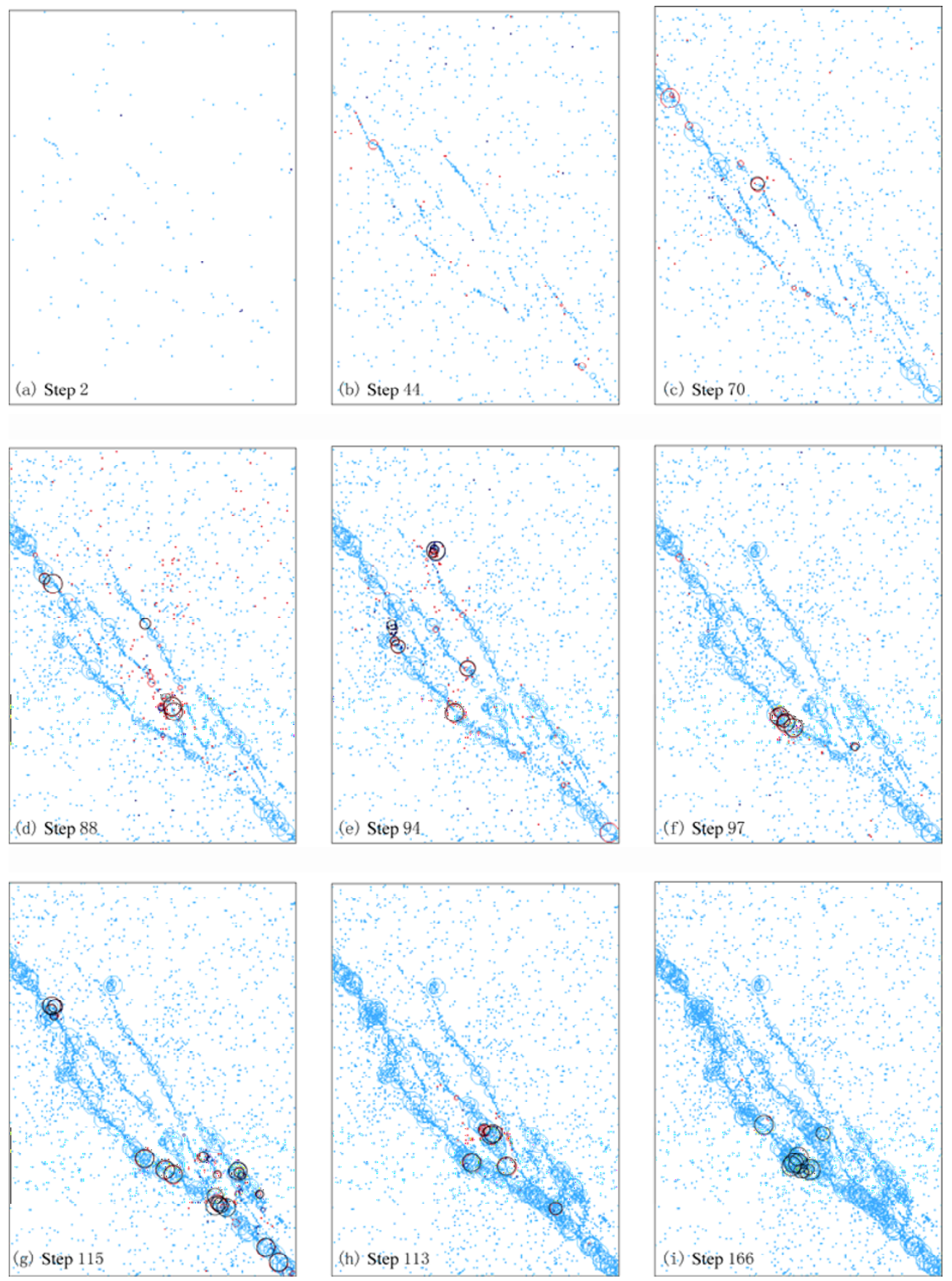

Figure 3 Simulated sequential plots showing the maps of seismic events in the earthquake evolution process. Stress interval for each plot is shown in Figure 2. Each circle represents one event and the diameter represents its relative magnitude. The green background circles gives all the event locations occurred before the current step. The red ones represent the events during the current step.

foreshocks. Our numerical simulation also provide negative evidence to the idea that earthquake can be well predicted based on the seismicity analysis. In earthquake prediction, this helpless is not just due to the highly sensitive nonlinear dependence of earthquake on unknown initial physical conditions, the statistical feature of seis- mic events and the fact that seismic activities always reflect information of the past behavior (failure already occurred) severely limit their predictability of forthcoming events. Although our modeling has reproduced some realistic spatio-temporal seismic patterns, we are unable to identify the model foreshocks in advance 
based on these spatial-temporal seismic patterns. This conclusion is also confirmed by our other simulations. However, the inherent unpredictability of individual earthquakes based on the statistical data of seismicity does not mean that earthquakes are unpredictable based on other strategy. The following section on SSS studies strongly supports the view of Crampin (1999) that stress monitoring on large scale may be a good strategy of earthquake prediction.

\section{Stress buildup, stress shadow and stress transfer (SSS)}

In a conventional experiment of rock failure, it is difficult if not impossible to measure the stress distribution throughout the failure process of a specimen. It is even more difficult to observe the stress field evolution in a natural earthquake cycle. However, the details of the development of such an essential stress feature regarding the failure process can be easily modeled numerically with RFPA code. Figure 4 shows the stress field evolution as a series of snapshots in time steps corresponding to the seismic maps, as illustrated in Figure 3. This figure shows clearly the simulated stress variation indicating the stress buildup, stress shadow and stress transfer (SSS).

\subsection{Stress buildup}

The controlling parameter responsible for the variation in stressing is the fault geometry, mechanical properties and the influence of the surrounding faults, as shown in Figure 1. Generally, two sites are found to be the candidates with priority as the highest stress buildup zone: (1) fault bridge or the area between en echelon faults (such as the area between faults " $a$ " and " $e$ ", or the area between faults " $e$ ", " $h$ " and " $i ")$; (2) obstacle or locked fault surfaces (such as the inflexion point in fault " $e$ "). A particular fault may be situated in a bright stress buildup zone or a stress shadow zone. A comparison between Figures 3 and 4 shows that, as observed in case studies (Deng and Sykes, 1997), most of the moderateto large-size seismic shocks can be identified to occur in regions predicted to be high stress buildup zones, i.e., bright zones as shown in Figure 4. Particularly, the locations of two mainshocks in the simulation are found to be predictable based on the stress-forecasting strategy. Figure 4f indicates the bright stress regions just before the mainshock. The mainshock with the highest energy release occurs in step 115 in the region marked with a red circle that is believed to be the most potential can- didate site to induce high-energy release rupture. As expected, the biggest shock occurs in the high stress buildup zone in step 115 and has affected greatly the stress re-distribution on the nearby faults. In Figure 4h, circle II marks the region of stress shadow induced by this mainshock. While circle I in Figure 4h (step 133) shows the state of high bright stress buildup just before the second mainshock that occurs in step 166 as shown in Figure 4i. A detailed description of the stress variation along the segment between the two mainshock sites has been displayed in Figure 5 with the first figure showing the stress buildup histories and the second one showing the stress states immediately before the two mainshocks.

The difference between the two rupture modes for the first and second mainshocks is that the first one is a coalescence rupture of intact rock between the junction region of fault " $e$ ", " $h$ ", " $i$ " and " $j$ ", while the second one is a rupture of the locked fault. We find that the coalescence rupture also occurred between the overlapped faults " $a$ " and " $e$ " shown in step 94 in Figure 4e. The coalescence rupture mode demonstrates an important feature of fault rupture, the so-called jump-stepover rupture that a detailed study on it is rare (Harris and Day, 1999). This failure mode has been observed in many natural earthquakes. For example, the 1992 Landers earthquake initiated on one fault, and then jumped across numerous geometrical boundaries to rupture through at least four more faults, becoming a M 7.5 event (Harris and Day, 1999). Although the probability of one earthquake cascading into another has been included in some hazard models (Jackson et al, 1995), what stops earthquakes after they begin rupturing is unknown (Harris and Day, 1999). Our simulation seems offering a reasonable explanation on this "stop" mechanism. As seen in step 70 (Figure 4c), the stress builds up around the bridge region between the faults " $a$ " and " $e$ ". At this step, the rock surrounding these tips of faults is strong so that the rupture cannot break into this "intact region". At step 88 (Figure 4d), stress is high enough to initial fractures in this intact region. The coalescence occurs in step 94 (Figure 4e), and this process continues until step 115 (Figure 4g) in which the stress in this region drops to a much lower level resulting a stress shadow, which moves the region away from its critical state, creating a period of relative quiescence. It is believed that this stress drop is partially due to the coalescence between the faults and partially due to the stress release caused by the first mainshock. 

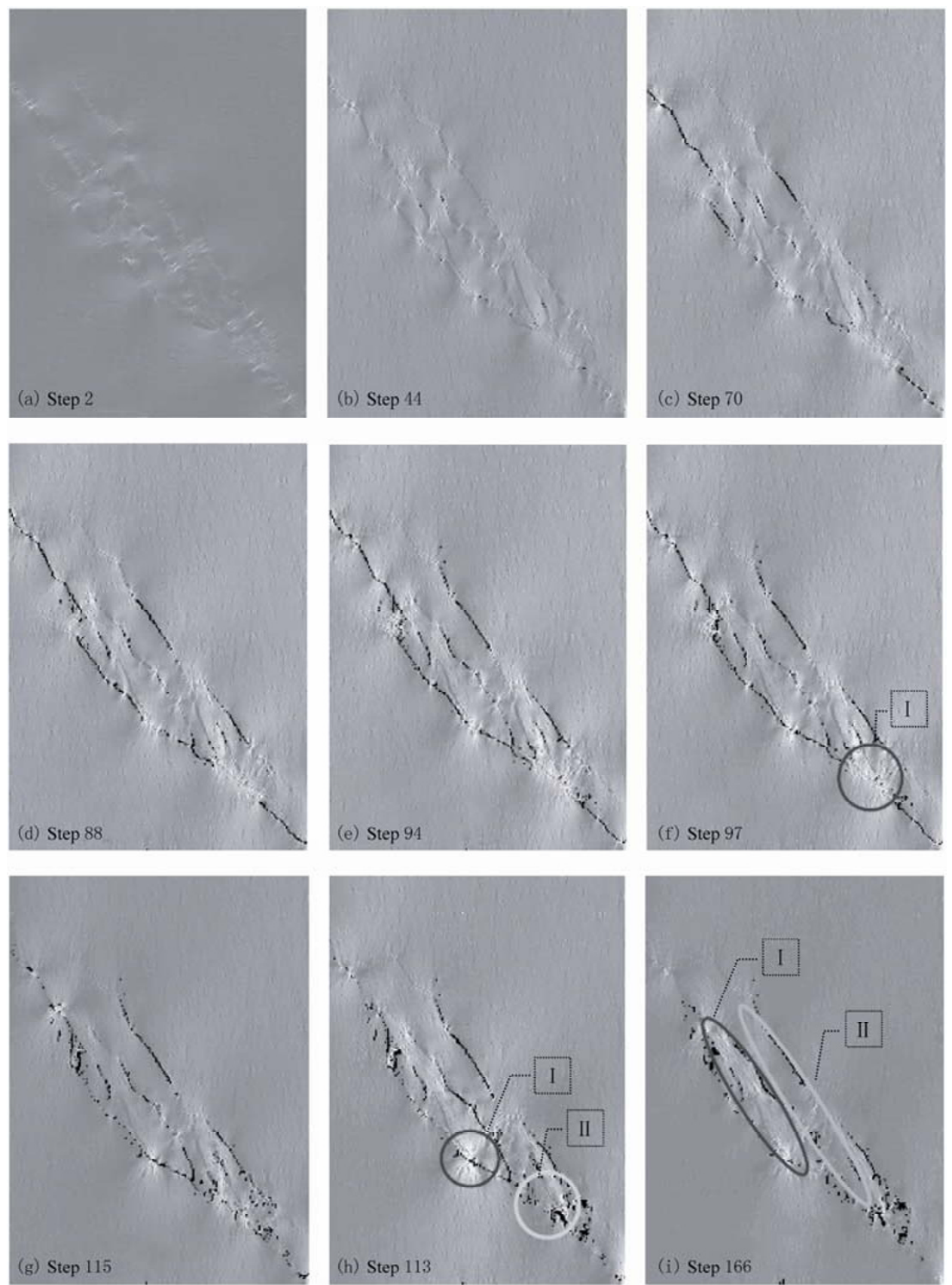

Figure 4 Simulated sequential plots showing the shear stress fields in the earthquake evolution process, corresponding to the maps of seismic events shown in Figure 3. The gray color grade represents the value of shear stress. This figure shows clearly the simulated stress variation indicating the stress buildup, stress shadow and the stress transfer (SSS). The circle I indicates the high bright stress region. The circle II indicates the mainshock induced stress shadow.

\subsection{Stress shadow}

Great earthquakes produce large stress shadow zones that will gradually narrowed with time as stress is re-accumulated by tectonic loading. As an aseismic sign, stress shadow is of great importance to provide some guides to long-term earthquake prediction. For example, the 1906 San Francisco earthquake produced a "shadow" in the static stress field that seemed to inhibit earthquakes for many years after the M7.9 event (Sammis and Bowman, 1999a). The above simulation has shown that in each step of the stress evolution modeling, most of the moderate to large shocks occurred in bright 
stress buildup zones, not in shadow zones. In the Nature earthquake debate, Scholz (1999) discussed stress shadows in the framework of SOC, and mentioned that this concept is equivalent to the "seismic gap" hypothesis. However, it should be noted that, if stress shadow is defined as stress released area due to large earthquake, to distinguish the difference between stress shadow and seismic gap is of great importance to earthquake predictions. Based on our simulation, although both being seismic quiescent, the stress level is high in site of seismic gap (expecting a large oncoming rupture), but low in site of stress shadow (induced by a previous rupture and no large rupture is expected). The two narrow elliptical zones shown in Figure 4i are two good examples to demonstrate the difference between stress shadow and seismic gap. The red elliptical zone is located between faults " $c$ " and " $f$ ", while the blue one is located between the faults " $b$ " and " $c$ ". Both are found to be located in aseismic zones during the previous loading steps. However, the stress field shown in Figure 4i shows that the red elliptical zone is located in the bright stress buildup zone, while the blue one is located in a stress shadow. As a result, the former will become more seismic active, while the latter will be free of seismic hazards. This numerical result may provide a positive answer to the question asked by Harris (1998), that is, stress shadow (regions when faults are relaxed) calculations can be used to estimate where and when future earthquakes will not occur.

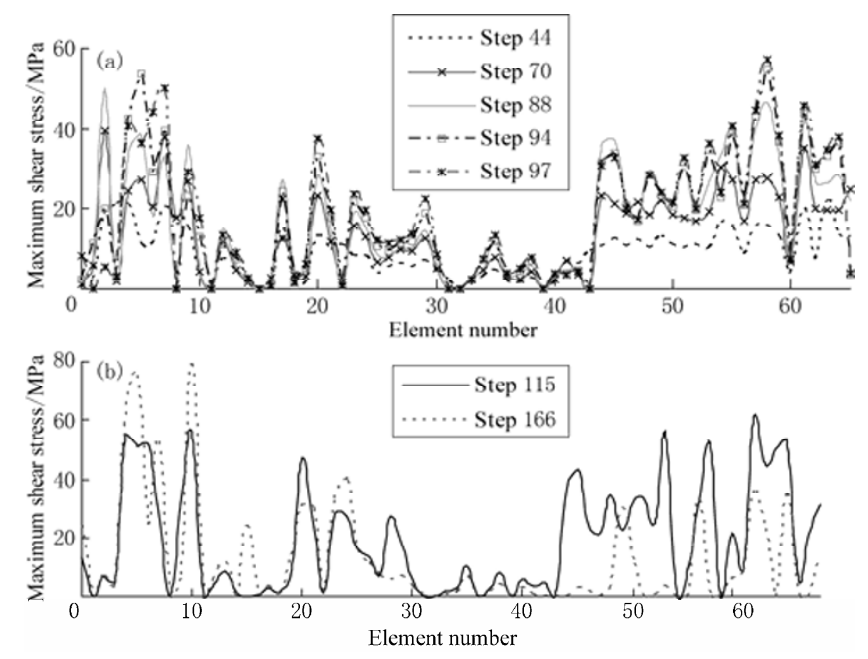

Figure 5 Simulated maximum shear stress history at several different time steps along the fault " $e$ " from the segments of second mainshock site to first mainshock site. The fluctuations in the stress level are caused partially from the heterogeneity setup in the original model and partially from the failure induced heterogeneity. (a) Stress buildup history before the first mainshock; (b) Stress until just before the step 115 mainshock and the step 166 mainshock.

\subsection{Stress transfer}

The numerical simulation also shows that ruptures may trigger other ruptures on nearby faults (clearly seen in Figures 3 and 4), which has been observed by many earthquake researchers (Harris and Simpson, 1996; Scholz, 1999). This suggests that warnings can be issued about those faults that have been brought closer to failure by the preceding nearby rupture, as shown in Figures $4 \mathrm{~h}$ and $4 \mathrm{i}$ that shortly after a large rupture the resulting stress increase is observed on nearby faults. Different from the stress buildup process related to the tectonic loading, the stress concentration forms immediately after the rupture or at the same time as the stress shadow forms. We found that unless these stress transfer induced stresses are high enough to trigger large rupture, they usually contribute to the aftershocks. It is worth to mention that, as pointed out by Deng and Sykes (1997), triggering does not mean that the coseismic stress buildup associated with one earthquake is enough to generate another earthquake in an originally stress-free location. As shown in Figure 4b, the stress at the site of the second mainshock is already close enough to failure that the first mainshock triggers the second one by introducing a $30 \%$ increase of the stress to move it into the failure regime.

\subsection{Stress evolution and earthquakes}

Our finding that most of the moderate to large modeled shocks occur in the bright high stress buildup regions (see next section) encourages us to continue our studies down to the more general topics of whether 
earthquake is a random SOC process that any small event has some probability of cascading into a large earthquake (Geller et al, 1977), anywhere at anytime (Scholz, 1999). At least from our study here, we think the answer is opposite. One of the main problems in standard SOC models comes from the absence of the fault geometry. Our simulation using the model containing the complex geometry of faults strongly supports the hypothesis (Jaumé 1999; Main, 1999) that earthquakes do not occur randomly in space and time. We have re-examined the seismic event map (Figure 3) and compared with the fault geometry in the original model (Figure 1), and find that most of the moderate to large seismic events contributing to the two seismic cycles (shown in Figure 2c) occur in two well defined regions within the fault system. To strengthen our finding, we have re-drawn the seismic location maps of the modeled epicenters with magnitude

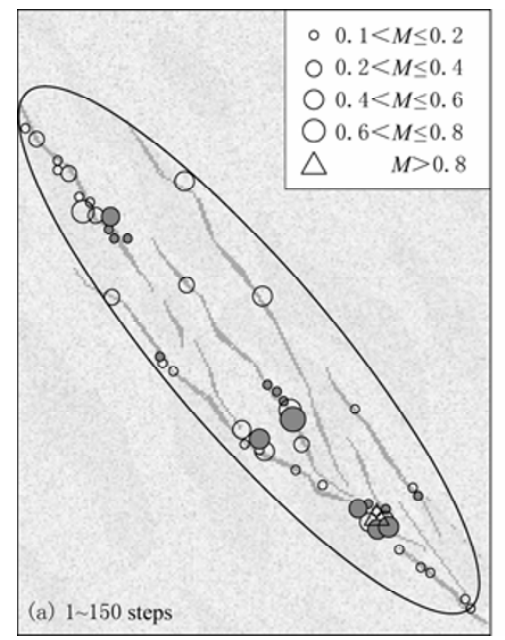

0.1 (normalized scale) and larger in Figure 6, with Figure $6 \mathrm{a}$ for the period 1 150 time steps (first earthquake cycle) and Figure $6 \mathrm{~b}$ for the period 151 200 steps (second cycle). In addition to the fact that all of the moderate to large shocks contributing to the foreshocks, mainshock and aftershocks in the first seismic cycle occur in a narrow elliptical region (coincidence with the fault zone), as shown in Figure 6a, we further find that all the moderate to large shocks, including foreshocks, mainshock and aftershocks, in the second seismic cycle occur in an elliptical region that is much smaller than that of the first seismic cycle, as shown in Figure 6b. Surprisingly, this second elliptical region is completely located inside the first elliptical region, which indicates that the size of seismic regions scales with the magnitude of the oncoming events and their locations are dependent on the seismic history and the original fault geometry

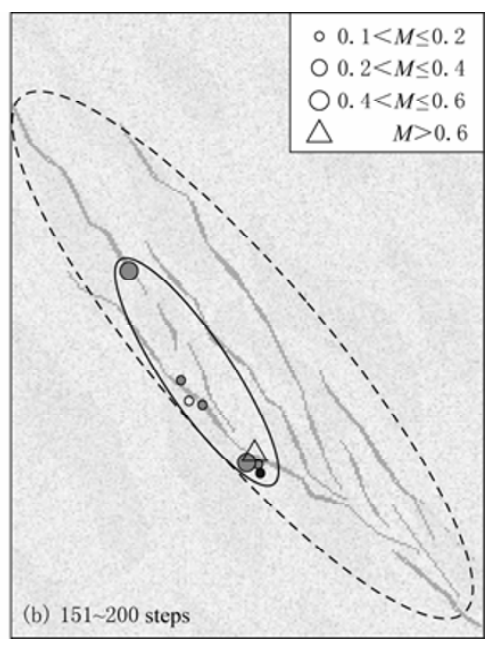

Figure 6 Simulated earthquake epicenters during two time periods. (a) Epicenters during the first earthquake cycle; (b) Epicenters during the second earthquake cycle. Open circles represent the foreshocks. Triangle represents the mainshock. Solid circles represent the aftershocks. $0<M<1$ represents the relative magnitude proportional to the seismic released energy.

The non-uniform distribution of stress field and the disturbance of principal stress orientation due to the complex geometry of fault system are other evidence to support the hypothesis (Jaumé and Sykes, 1999; Main 1999) that earthquakes do not occur randomly in space and time. If the crust is assumed as a random system, it is required that the tectonic stress is near failure everywhere and at all times, and that the stress drops are small, depleting the local elastic energy available for further ruptures only to an insignificant degree (Wyss, 1997). But our modeling of stress distribution leading up to and following mainshocks suggests otherwise. For example, the modeled first mainshock was anticipated on the basis of observed stress buildup (Figure 4). Over the loading steps prior to this shock, shear stress of 60 MPa accumulates, and during the rupture most of stress is released (Figure 4b). Figure 7 shows that within the fault zone areas the modeled principal stress orientation varies locally and large compressive or tensile stresses with disordered orientation are observed. Our modeling has shown that the seismic activity is associated with this local stress disturbance. In other regions where the principal stresses are oriented in the boundary loading direction little disturbance of stress orientation is found, indicating that these are inactive area in seismicity. 


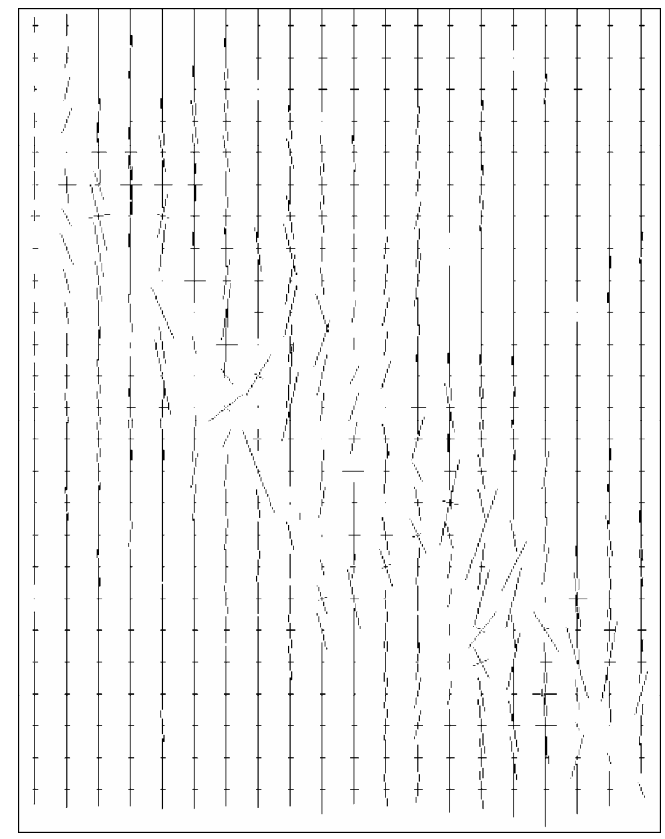

Figure 7 Simulated principal stress crosses. These crosses represent the horizontal stress field disturbance due to faulting in time step 88 .

\section{Conclusions}

Through a physics-based numerical model, we have intuitively demonstrated that the synthetic earthquake history, including the stress evolution and associated seismicity, can be generated to resemble in great details the observed earthquake phenomena. The modeled result, that most of the moderate to large shocks in the simulation occur in bright stress buildup regions (not in shadow regions), suggests that monitoring of stress field variation can provide important guidance to the medium- and long-term earthquake prediction in terms of SSS. This approach allows us to place the understanding of statistical features of seismicity on a firmer physical footing that a great earthquake can change the stress distribution and trigger (due to stress buildup) or shut off (due to stress shadow) moderate to large seismic events in different regions depending on the seismic history, the geometrical and mechanical properties of the fault system. One of the most important conclusions is that earthquakes are predictable if the stress buildup, stress shadow and stress transfer (SSS) can be well monitored. This numerical modeling with complex geometry of fault system and medium heterogeneity being considered provides a useful tool to improve our understanding of the correlation between the stress field variation and the data from GPS or InSAR observations.
Such modeling may open a number of possibilities for future research in earthquake dynamics and earthquake prediction based on the continuously developing stress or deformation monitoring techniques.

Acknowledgements This study is supported by the National Key Basic Research Science Foundation (No. 2007CB209400), the National Natural Science Foundation of China (No. 40638040, 10672028) and the Chinese Postdoctoral Science Foundation (No. 20070421048).

\section{References}

Abraham F F, Brodbeck D, Rafey R A and Rudge W E (1994). Instability dynamics of fracture: A computer simulation investigation. Phys Rev Lett 73: 272-276.

Brace W F and Byerlee J D (1966). Stick slip as a mechanism for earthquakes. Science 153: 990-992.

Bufe C G and Varnes D J (1993). Predictive modeling of the seismic cycle of the greater San Francisco Bay region. $J$ Geophys Res 98(B6): 9 8719883.

Chen Y Q, Zhang G B, Ding X L and Li Z L (2000). Monitoring earth surface deformations with INSAR technology: Principle and some critical issues. J Geospatial Eng 2(1): 3-21.

Crampin S (1999). Stress-forecasting: An apparently viable third strategy. Nature, http://helix.nature.Com/debates/earthquake/, April 1999.

Deng J and Sykes L R (1997). Evolution of the stress field in southern California and triggering of moderate-size earthquakes. $J$ Geophys Res 102: 9 859-9 886.

Geller R J, Jackson D D, Kagan Y Y and Mulargia F (1977). Earthquake cannot be predicted. Science 275: 1616-1 617.

Harris R A and Day S M (1999). Dynamic 3D simulations of earthquakes on en echelon faults. Geophys Res Lett 26: 2 089-2 092.

Harris R A and Simpson R W (1996). In the shadow of 1857: The effect of the great Ft. Tejon earthquake on subsequent earthquakes in southern California. Geophys Res Lett 23: 229-232.

Harris R A (1998). Introduction to special section: Stress triggers, stress shadows, and implications for seismic hazard. J Geophys Res 103: 24 347-24 358.

Heimpel M (1997). Critical behavior and the evolution of fault strength during earthquake cycles. Nature 388: 865-868.

Huang Y, Saleur H, Sammis C G and Sornette D (1998). Precursors, aftershocks, criticality and self-organized criticality. Europhysics Letters 41(1): 43-48.

Jackson D D, Aki K, Cornell C A, Dieterich J H, Henyey T L, Mahdyiar M, Schwartz D and Ward S N (1995). Seismic hazards in Southern California: Probable earthquakes, 1994 to 2024. Bull Seism Soc Amer, 85(2): 379-439.

Jaumé S C and Sykes L R (1999). Evolving towards a critical point: A review of accelerating moment/energy release prior to large and great earthquakes. Pure Appl Geophys 155: 270-306.

Liu Z, Myer L R and Cook N W G (1994). Numerical simulation of the effect of heterogeneities on macro behavior of granular materials. In: Siriwardane $\mathrm{Z}$ ed. Computer Method and Advanced in Geomechanics. A. A. Balkema, Rotterdam, 611-616.

Main I (1999). Is the reliable prediction of individual earthquakes a realistic scientific goal? Nature http://helix.nature.Com/debates/earthquake/, April 1999.

Marder M and Liu X M (1993). Instability in lattice fracture. Phys Rev Lett 71: $2414-2420$.

Mogi K (1969). Some features of recent seismic activity in and near Japan (2). Activity before and after great earthquakes. Bull Earthq Res Inst Univ Tokyo 47: 395-417. 
Peltzer G, Crampe F and Goffrey K (1999). Evidence of nonlinear elasticity of the crust from the $M_{\mathrm{W}} 7.6$ Manyi (Tibet) Earthquake. Science 268: 272-275.

Reilinger R E, Ergintav S, Burgmann R, McClusky S, Lenk O, Barka A, Gurkan O, Hearn L, Feigl K L, Cakmak R, Aktug B, Ozener H and Toksoz M N (2000). Coseismic and postseismic fault slip for the 17 August 1999, $M=7.5$, Izmit, Turkey Earthquake. Science 289(5484): 1 519-1 524.

Bauman D and Sammis Ch (1999). A case for intermediate-term Earthquake Prediction: Don't throw the baby out with the bath water. Nature debates: Is the reliable prediction of individual earthquake a realistic scientific goal, 25th February - 8th April.

Sammis C G and Smith S W (1999b). Seismic cycles and the evolution of stress correlation in cellular automation models of finite fault networks. Pure
Appl Geophys 155: 307-334.

Scholz C H (1999). Earthquake prediction: Feasible and useful? Nature, http://helix.nature.Com/debates/earthquake/, April 1999.

Scholz C H (1998). Earthquakes and friction laws. Nature 391: 37-42.

Scott D R (1996). Seismicity and stress rotation in a granular model of brittle crust. Nature 381: 592-595.

Tang C A (1997). Numerical simulation of progressive rock failure and associated seismicity. Int J Rock Mech Min Sci 34: 249-262.

Wyss M (1997). Cannot earthquakes be predicted? Science 278: 487.

Zapperi S, Vespignani A and Stanley H E (1997). Plasticity and avalanche behavior in microfracturing phenomena. Nature 388: 658-660. 